Technical note

\title{
Cobalt thin films on gold: A new reference material for the quantification of cobalt phthalocyanine and cobalt porphyrin modified gold electrodes with synchrotron radiation micro X-ray fluorescence spectroscopy
}

\author{
Annemie Adriaens ${ }^{\mathrm{a}, *}$, Karolien De Wael ${ }^{\mathrm{a}, 1}$, David Bogaert ${ }^{\mathrm{a}}$, Hans Buschop ${ }^{\mathrm{a}}$, Tom Schoonjans ${ }^{\mathrm{a}}$, \\ Bart Vekemans $^{a}$, Diederik Depla ${ }^{\mathrm{b}}$, Laszlo Vincze ${ }^{\mathrm{a}}$ \\ a Ghent University, Department of Analytical Chemistry, Krijgslaan 281 S12, B-9000 Ghent, Belgium \\ ${ }^{\mathrm{b}}$ Ghent University, Department of Solid State Science, Krijgslaan 281 S1, B-9000 Ghent, Belgium
}

\section{A R T I C L E I N F O}

Article history:

Received 14 March 2008

Accepted 14 June 2008

Available online 28 June 2008

Keywords:

Reference material

Cobalt

Gold surface

SR micro-XRF

\section{A B S T R A C T}

New reference materials consisting of cobalt thin films on gold were prepared by sputter deposition. The thickness and homogeneity of the films were characterized using synchrotron radiation micro-XRF. The samples can be used as reference materials to quantify cobalt phthalocyanine and cobalt porphyrin modified gold electrodes which have been analyzed with synchrotron radiation micro-XRF.

(c) 2008 Elsevier B.V. All rights reserved.

\section{Introduction}

Synchrotron radiation micro X-ray fluorescence spectroscopy (SR micro-XRF) offers an excellent capability to perform elemental mapping of elements in very low concentrations. In our work, for instance, the technique has been successfully exploited for quantifying the surface coverage of gold electrodes modified with a cobalt (II) tetrasulfonated phthalocyanine tetrasodium salt (CoTSPc) thin layer [1-3]. These kinds of modified electrodes often exhibit electrocatalytic properties, which allow specific electrochemical reactions to occur with much lower activation energies. Together with the metalloporphyrin systems, they have therefore attracted a wide research interest and are considered as the first step in the development of new electrochemical sensors [4-6].

The cobalt surface concentrations of the samples analyzed typically range between 0.05 and $3.5 \mu \mathrm{cm}^{-2}$. The major shortcoming in their quantification up to now has been the lack of suitable reference materials. The latter has resulted in needing to perform timeconsuming calculations based on the fundamental parameter method for each measurement [7].

In this work we have tried to counter this practical problem by preparing new standard materials which have the same design as the modified electrodes, namely a cobalt thin layer onto a gold substrate.

\footnotetext{
* Corresponding author. Tel.: +32 9264 4826; fax: +32 92644960 . E-mail address: annemie.adriaens@ugent.be (A. Adriaens).

1 Postdoctoral Fellow of the Research Foundation - Flanders (Belgium).
}

The samples have been prepared by sputter depositing a cobalt layer of various thicknesses (between 10 and $100 \mathrm{~nm}$ ) on a gold substrate.

This paper describes the preparation and the characterization of the new reference materials.

\section{Experimental}

\subsection{Preparation of the reference materials}

The commonly used gold electrodes were imitated by encapsulating a piece of gold wire (diameter $1 \mathrm{~mm}$ ) in epoxy resin (diameter $8 \mathrm{~mm}$ ) so that only the top surface of the gold wire was exposed. The

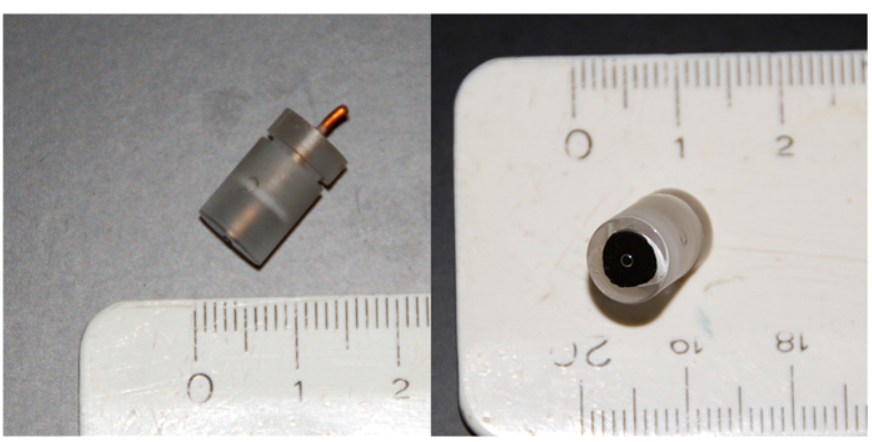

Fig. 1. Photograph of one of the prepared samples. The other three are identical. 


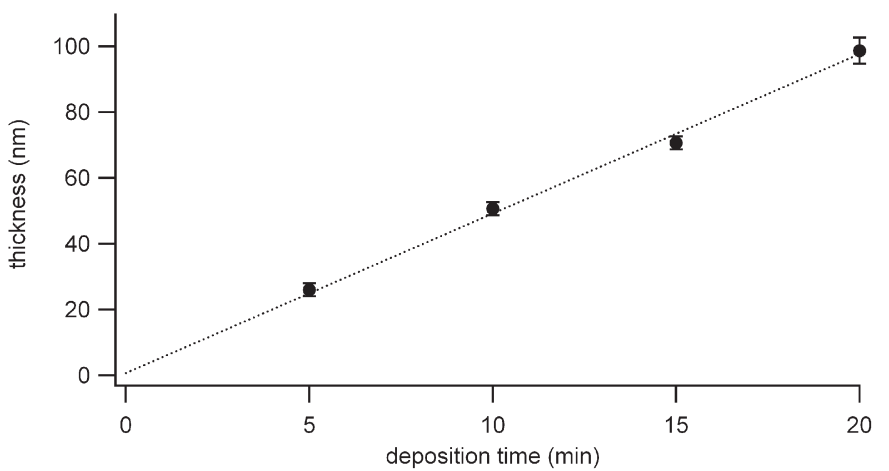

Fig. 2. Thickness of cobalt layers on glass substrates as a function of the deposition time. Deposition conditions: constant current $(0.1 \mathrm{~A})$ and constant argon pressure $(0.3 \mathrm{~Pa})$ The circles are the measured data. The solid line represents a linear fit through the data. Correlation coefficient is 0.996 .

wire exits the epoxy resin at the back in order to make an electrical connection, in case one likes to perform electrochemical measurements. Fig. 1 shows a set of photographs of one of the samples. The other ones are identical in construction.

A thin layer of cobalt (spot diameter $5.5 \mathrm{~mm}$ ) was deposited on the samples using magnetron sputter deposition. The home built magnetron sputter deposition system consists of a stainless steel chamber which is pumped by a turbo molecular pump with a rotary pump as back up (base pressure lower than $7 \times 10^{-4} \mathrm{~Pa}$ ). The system consists of a water cooled copper cathode on which a two inch $0.5 \mathrm{~mm}$ thick cobalt target (Kurt Lesker, 99.99\%) was clamped. The argon discharge is ignited using a DC power supply (Hüttinger 1500 DC). The depositions were performed at constant current $(0.1 \mathrm{~A})$ and constant argon pressure $(0.3 \mathrm{~Pa}, 30 \mathrm{sccm})$. Keeping the deposition parameters constant (base pressure $4.5 \times 10^{-4} \mathrm{~Pa}$ and discharge voltage $365.2 \mathrm{~V}$ ), the thickness of the deposited layers was controlled by exposing the gold electrodes to the magnetron for a given time (respectively 1'50", 3'58", 10'10" and 20'29"). The deposition rate was measured by depositing cobalt on glass substrates for a given time and measuring the thickness using a profilometer (Taylor Hobson Talystep). In total four samples were made with a cobalt layer thickness between 10 and $100 \mathrm{~nm}$.

\subsection{Characterization of the reference materials}

Scanning micro SR-XRF experiments were performed at beam line L of the DORIS-III storage ring, HASYLAB (Hamburg, Germany) [8]. This beamline is dedicated to micro-XRF experiments using either white or monochromatic bending magnet excitation and mono- or polycapillary focusing, with routinely available beam sizes of 5-30 $\mu \mathrm{m}$.

The primary X-ray beam is generated by a $1.2 \mathrm{~T}$ bending magnet source which, given the machine energy of $4.465 \mathrm{GeV}$, provides a polychromatic spectral distribution with a critical energy of $16.6 \mathrm{keV}$. After initial low-energy filtering and collimation, the beam is monochromatized by a $\operatorname{Si}(111)$ fixed-exit monochromator $\left(\triangle E / E \approx 10^{-4}\right)$ and further collimated by a motorized cross-slit system. During these experiments, the beam was focused by a monolithic polycapillary halflens (X-ray Optical Systems Inc., USA), designed specifically for parallel primary beams hence suitable for the focusing of low-divergence synchrotron beams. A microbeam size of about $20 \mu \mathrm{m}$ (FWHM) was obtained at the excitation energy range of 7.7-8.2 keV, which was used to excite selectively the $\mathrm{Co}-\mathrm{K}_{\alpha, \beta}$ fluorescent-lines without exciting $\mathrm{Au}-\mathrm{L}$ lines from the (spectroscopically infinitely thick) gold substrate. The nominal working distance of the polycapillary optic was $5 \mathrm{~mm}$.

For each sample $31 \times 31$ pixel maps were recorded with typically $20 \mu \mathrm{m}$ step-size, using a data collection time of $10 \mathrm{~s}$ per data point. Quantification of the cobalt surface involved transferring the individual pixel spectra into a sum spectrum using MicroXRF2 software and converting the measured fluorescent intensities to a concentration value including corrections for background and line overlap [9]. The non-linear least square fitting software AXIL [9] was used to determine the net-peak intensities of the $\mathrm{Co}-\mathrm{K}_{\alpha}$ and the background below this peak. A pure thin Co foil (thickness $10 \mu \mathrm{m}$, purity 99.9\%) from Goodfellow Inc. was used as reference material. The actual concentrations were calculated using the following equation, which is based on the fundamental parameter method [7]:

$S_{u}=\frac{I_{u}}{I_{0, u}} \frac{I_{0, s}}{I_{S}} \rho_{s} \cdot T_{S} \cdot A_{\text {corr }, s}$

where $S_{u}$ is the surface concentration of cobalt on the unknown material $\left(\mathrm{g} \mathrm{cm}^{-2}\right) ; I_{u / s}$ the measured intensity of the cobalt signal per time unit (counts $\mathrm{s}^{-1}$ ) on the unknown sample/standard material; $I_{0, u / s}$ the signal per time unit measured by the ionisation chamber for the unknown

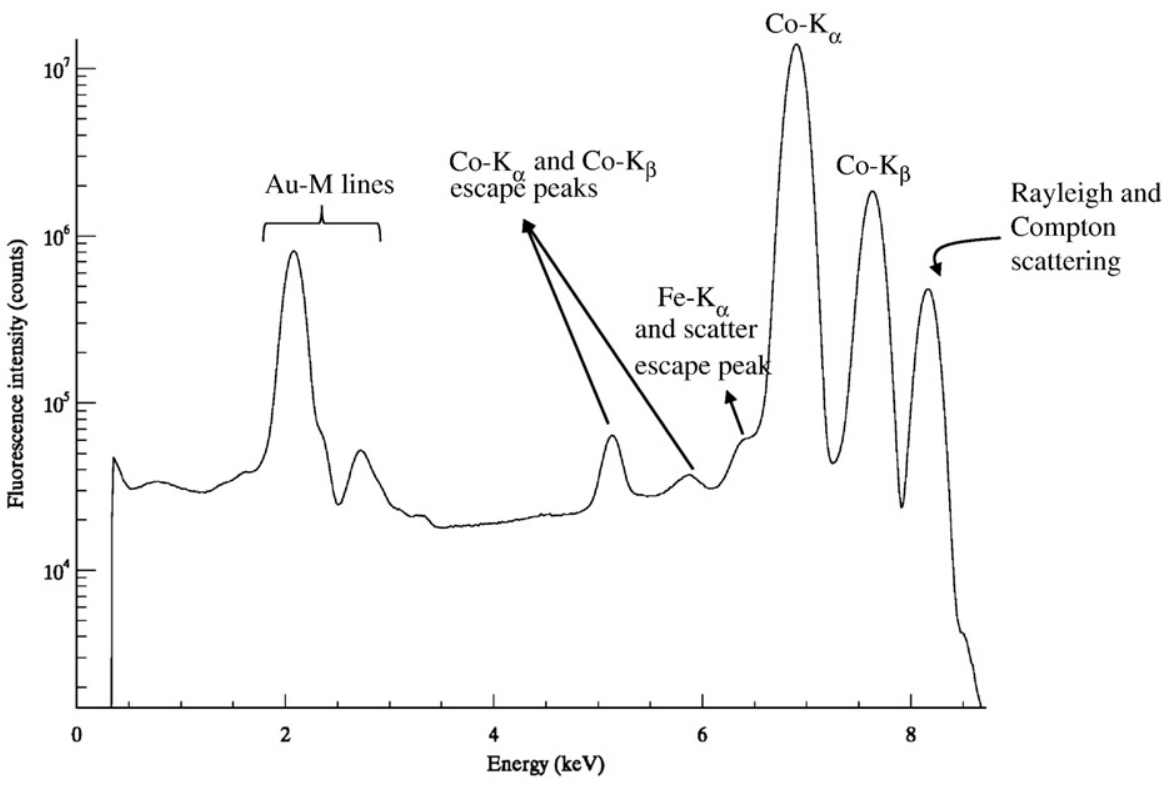

Fig. 3. Sum spectrum of the $31 \times 31$-pixel analyzed area of the $100 \mathrm{~nm}$ cobalt sample. 
Table 1

Cobalt surface concentration $\left(\mu \mathrm{g} / \mathrm{cm}^{2}\right)$ and thickness cobalt layer $(\mathrm{nm})$ for each of the four materials as determined by SR micro-XRF and the fundamental parameter method

\begin{tabular}{llcc}
\hline $\begin{array}{l}\text { Intended thickness Co } \\
\text { layer }\end{array}$ & $\begin{array}{l}\text { Co surface concentration } \\
\left(\mu \mathrm{g} / \mathrm{cm}^{2}\right)\end{array}$ & $\begin{array}{l}\text { Thickness cobalt } \\
\text { layer }(\mathrm{nm})\end{array}$ & $\begin{array}{l}\text { Deviation } \\
(\%)\end{array}$ \\
\hline $10 \mathrm{~nm}$ & 9.70 & 10.87 & 8.73 \\
$20 \mathrm{~nm}$ & 16.96 & 19.02 & -4.92 \\
$50 \mathrm{~nm}$ & 45.59 & 51.11 & 2.23 \\
$100 \mathrm{~nm}$ & 93.62 & 104.96 & 4.96 \\
\hline
\end{tabular}

sample/standard reference material; $\rho_{s}$ the density of metallic cobalt ( $\left.=8.92 \mathrm{~g} \mathrm{~cm}^{-3}[10]\right) ; T_{s}$ the thickness of the cobalt reference material (as cited above) and $A_{\text {corr,s }}$ the absorption correction factor for the cobalt reference material $(=0.20948[9,11])$.

Additionally the level of uniformity was determined by processing the data according to the strategy of Kempenaers et al. [12-13]. The latter involves the following steps. The Co- $\mathrm{K}_{\alpha}$ intensity is normalized to the signal of the Au-M line, after which its distribution is examined by constructing a histogram. A Gauss curve is fitted to the histogram which allows one to determine the mean, the standard deviation and the relative standard deviation.

\section{Results and discussion}

Fig. 2 shows the measured thickness of cobalt layers on glass as a function of deposition time. The symbols correspond to the measured data. The solid line represents a linear fit through the data. Based on this fit, the required deposition time was calculated in order to make the four reference materials with respectively 10, 20, 50 and $100 \mathrm{~nm}$ thickness. The error on the thickness was estimated to be between $4 \mathrm{~nm}$ (thinnest layers) and $8 \mathrm{~nm}$ (thickest layers). The latter is defined by the thickness measurements (profilometry) and the experimental error on the deposition parameters (pressure, discharge current and the time measurement).

A typical SR micro-XRF sum spectrum $(31 \times 31$ pixels) for the sample containing an estimated thickness of $100 \mathrm{~nm}$ cobalt is shown in Fig. 3. The sum spectra for the other samples are very similar. Apart from the clear presence of cobalt and gold, the spectrum shows evidence for the presence of iron. Its signal overlaps with the scatter escape peak. The presence of iron can be considered as contamination and originates possibly from a number of sources, including the magnetron sputter deposition system as well as the SR microXRF set-up and the presence of dust particles in the ambient environment.

Table 1 lists the surface concentration and the thickness of the cobalt layers as determined by SR micro-XRF. In addition the deviation between the measured and the intended thickness is listed. Results show that for all four samples the measured thickness approaches the intended thickness very well. Except for the $10 \mathrm{~nm}$ sample, all samples lie within a 5\% deviation (the deviation for the $50 \mathrm{~nm}$ sample is even of the order of $2.5 \%$ ). The $10 \mathrm{~nm}$ sample shows the largest deviation: around 9\%. Nevertheless the deviation lies within the range defined by the thickness
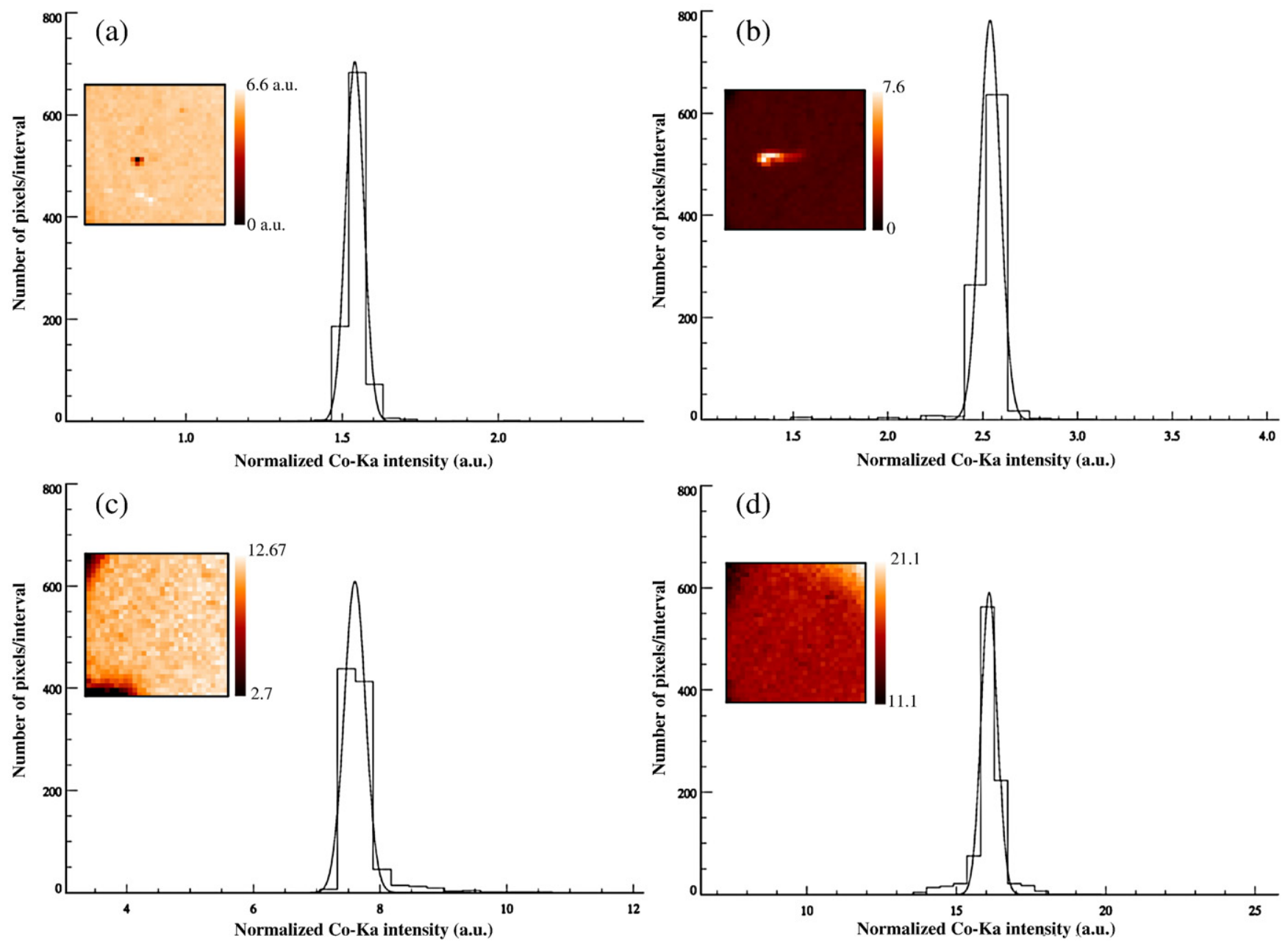

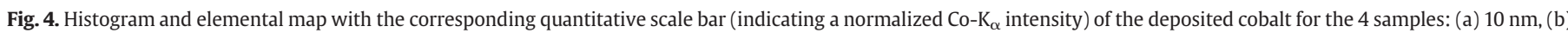
$20 \mathrm{~nm}$, (c) $50 \mathrm{~nm}$ and (d) $100 \mathrm{~nm}$. 


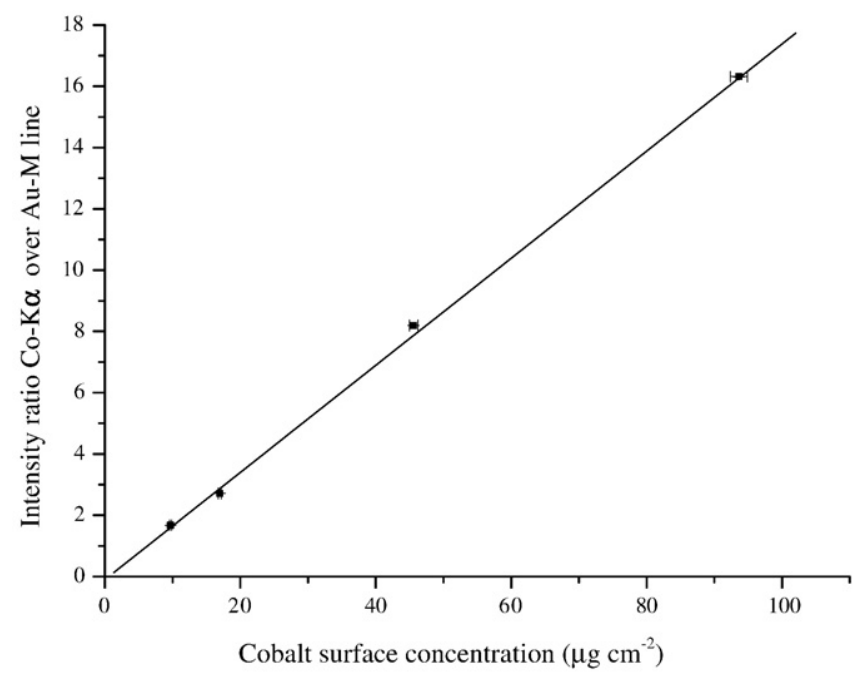

Fig. 5. The detected $\mathrm{Co}_{0} \mathrm{~K}_{\alpha}$ signal after normalization with the gold $\mathrm{M}$ line as a function of the cobalt surface concentration.

measurements and the experimental error on the deposition parameters (as cited above).

The elemental map and the corresponding intensity distribution (histogram) of the detected $\mathrm{Co}_{0} \mathrm{~K}_{\alpha}$ signal after normalization with the gold $\mathrm{M}$ line is shown in Fig. 4 for the four samples. The colours of the map are scaled between the minimum and the maximum value measured across the entire image. The latter implies that one artefact pixel of extremely high or low concentration can change the entire scale. Since each measurement showed at least 1 high or low extremity, we opted to correct for these outliers to obtain figures that are not all white or black (low and high extremity respectively). The correction was done by substituting any value higher or lower than the threshold by the value of the threshold ( 5 arbitrary units). These corrections, however, were not included in the calculations so as not to make any false conclusion about the homogeneity. The histograms show an increasing normalized $\mathrm{Co}-\mathrm{K} \alpha$ intensity of the cobalt signal with deposition time. The relative standard deviation is for all samples of the same order (ca. 1.5\%), demonstrating a reproducible and homogeneous production process of the standard materials.

The relationship between the detected $\mathrm{Co}_{0} \mathrm{~K}_{\alpha}$ signal after normalization with the gold $\mathrm{M}$ line and the cobalt surface concentration as measured by the fundamental parameter method is shown in Fig. 5. The symbols represent the actual data points. The solid line has been fitted through the points and shows a very good correlation between the two parameters, which implies that the data can be used as a calibration curve for future quantifications of cobalt phthalocyanine and cobalt porphyrin thin films on gold.

\section{Conclusions}

In this work we have prepared a set of new reference materials which can be used to quantify cobalt phthalocyanine and cobalt porphyrin films on gold electrodes using SR micro-XRF. The samples were prepared by sputter depositing cobalt films of various thicknesses (between 10 and $100 \mathrm{~nm}$ ) on a gold substrate. Characterization of the films was done using SR micro-XRF. For all four samples the measured thickness approaches the intended thickness very well. The surface concentrations were calculated using the fundamental parameter method and show a very good correlation with the detected cobalt signal. The heterogeneity of all samples is below $1.5 \%$.

\section{Acknowledgments}

This work was supported by the European Community - Research Infrastructure Action under the FP6 "Structuring the European Research Area" Programme (through the Integrated Infrastructure Initiative "Integrating Activity on Synchrotron and Free Electron Laser Science").

\section{References}

[1] K. Peeters, K. De Wael, A. Adriaens, G. Falkenberg, L. Vincze, Non-destructive characterisation of the CoTSPc deposition on gold electrodes by means of synchrotron X-ray fluorescence, Electrochemistry Communications 7 (2005) 1157-1162.

[2] K. Peeters, K. De Wael, L. Vincze, A. Adriaens, Comparison of different surface modification techniques for electrodes by means of electrochemistry and micro synchrotron radiation X-ray fluorescence. Dimerization of cobalt(II) tetrasulfonated phthalocyanine and its influence on the electrodeposition on gold surfaces, Analytical Chemistry 77 (17) (2005) 5512-5519.

[3] K. Peeters, K. De Wael, A. Adriaens, G. Falkenberg, L. Vincze, Quantitative synchrotron micro-XRF study of CoTSPc and CuTSPc thin-films deposited on gold by electrodeposition, Journal of Atomic Analytical Spectroscopy 22 (5) (2007) 493-501.

[4] R.W. Murray, A.G. Ewing, R. Durst, Chemically modified electrodes - molecular design for electroanalysis, Anal. Chem. 59 (5) (1987) 379-390.

[5] J. Wang, Modified electrodes for electrochemical sensors, Electroanalysis 3 (4-5) (1991) 469-471.

[6] D.W.M. Arrigan, Tutorial review. Voltammetric determination of trace metals and organics after accumulation at modified electrodes, Analyst 199 (1994) 1953-1966.

[7] A.A. Marckowicz, R. Van Grieken, Quantification in XRF analysis of intermediate thickness samples, in: R. Van Grieken, A.A. Marcowicz (Eds.), Handbook of X-ray Spectrometry, Marcel Dekker Inc, New York, 2001, pp. 407-431.

[8] G. Falkenberg, O. Clauss, A. Swiderski, T. Tschentscher, Upgrade of the X-ray fluorescence beam line at HASYLAB/DESY, X-ray Spectrom. 30 (2001) 170-173.

[9] B. Vekemans, K. Janssens, L. Vincze, F. Adams, P. Van Espen, Analysis of X-ray spectra by iterative least-squares (AXIL) - new developments, X-ray Spectrom. 23 (1994) 278-285

[10] The Merck Index12th Edition, Merck Research Laboratories, Whitehouse Station, New Jersey, 1996.

[11] A. Brunetti, M. Sanchez del Rio, B. Golosio, A. Simionovici, A. Somogyi, A library for $\mathrm{X}$-ray-matter interaction cross section for X-ray fluorescence applications, Spectrochim. Acta Part B 59 (2004) 1725-1731.

[12] L. Kempenaers, L. Vincze, K. Janssens, The use of synchrotron micro-XRF for characterization of the micro-heterogeneity of heavy metals in low- $Z$ reference materials, Spectrochim. Acta Part B 55 (2000) 651-669.

[13] L. Kempenaers, K. Janssens, L. Vincze, B. Vekemans, A. Somogyi, M. Drakopolous, A. Simionivici, F. Adams, A Monte Carlo model for studying the microheterogeneity of trace elements in reference materials by means of synchrotron microscopic X-ray fluorescence, Anal. Chem. 74 (19) (2002) 5017-5026. 